\title{
Three Human Chromosomal Autoantigens Are Recognized by Sera from Patients with Anti-centromere Antibodies
}

William Earnshaw, Bonnie Bordwell, Cathy Marino, and Naomi Rothfield

Department of Cell Biology and Anatomy, Johns Hopkins University School of Medicine, Baltimore, Maryland 21205; and Division of Rheumatic Diseases, Department of Medicine, University of Connecticut School of Medicine, Farmington, Connecticut 06032

\begin{abstract}
We have identified 39 individuals with anti-centromere antibodies (ACA) in our patient population, all of whom have Raynaud's syndrome or disease. We have used sera from the ACA-positive patients and from 123 controls (22 normal individuals and 101 additional patients with either Raynaud's disease or Raynaud's syndrome plus an associated connective tissue disease) to screen the proteins of highly purified human (HeLa) mitotic chromosomes by sodium dodecyl sulfate polyacrylamide gel electrophoresis and immunoblotting. Three antigens were recognized by the sera from the ACA-positive patients. These were centromere protein (CENP)-B (80,000 mol wt-recognized by all ACApositive sera), CENP-A (17,000 mol wt-recognized by 38 of 39 ACA-positive sera), and CENP-C (140,000 mol wt-recognized by 37 of 39 ACA-positive sera). None of these antigens were recognized by any of the 123 control sera, although binding was occasionally seen to other chromosomal antigens. Therefore the ACA response is highly uniform in our patient population. Antibody to CENP-B shows a $\mathbf{1 0 0 \%}$ correlation with anti-centromere staining by indirect immunofluorescence.
\end{abstract}

\section{Introduction}

Anti-centromere antibodies (ACA) ${ }^{1}$ are present in sera of certain patients with scleroderma, particularly the calcinosis, Raynaud's phenomenon, esophageal dysmotility, sclerodactyly, and telangiectasia (CREST) variant (1-5). ACA also have been found infrequently in some individuals with rheumatoid arthritis (6), pulmonary fibrosis (7), and primary biliary cirrhosis associated with CREST $(8,9)$. In addition, ACA have been noted in serum from patients with Raynaud's phenomenon associated with symptoms of connective tissue diseases other than sclerodermaCREST (4). We have previously described the presence of ACA in a patient with Raynaud's disease and in the serum of a patient with isolated Raynaud's syndrome $9 \mathrm{yr}$ before the development of CREST (10).

Address reprint requests to Dr. Earnshaw, Department of Cell Biology and Anatomy, Johns Hopkins University School of Medicine, Baltimore, MD 21205.

Received for publication 10 January 1985 and in revised form 21 October 1985

1. Abbreviations used in this paper: ACA, anti-centromere antibodies; ANA, antinuclear antibodies; ARA, American Rheumatism Association; CENP(s), centromere protein(s); CREST: C, calcinosis, R, Raynaud's phenomenon, E, esophageal dysmotility, S, sclerodactyly, T, telangiectasia; RA, rheumatoid arthritis; SDS-PAGE, sodium dodecyl sulfate polyacrylamide gel electrophoresis; SLE, systemic lupus erythematosus.

J. Clin. Invest.

(C) The American Society for Clinical Investigation, Inc.

0021-9738/86/02/0426/05 \$1.00

Volume 77, February 1986, 426-430
Despite an earlier report that digestion with DNase 1 destroyed the centromere antigen (1), a number of the antigens are proteins which can be identified by sodium dodecyl sulfate polyacrylamide gel electrophoresis (SDS-PAGE) and subsequent immunoblotting. This technique has led to identification of the following human antigens: apparent molecular weight $\left(M_{\mathrm{r}}\right)$ $14,000,20,000,23,000,34,000(11) ; M_{\mathrm{r}} 77,000,110,000$ (12); $M_{\mathrm{r}} 17,000,21,000,24,000,80,000,140,000(13) ; M_{\mathrm{r}} 19,500$, 23,000, 26,000 (14); $M_{\mathrm{r}} 33,000$ (15); and $M_{\mathrm{r}} 70,000$ (16).

Most investigators agree that one antigen is a protein of $M_{\mathrm{r}}$ 14-19,000. We refer to this as centromere protein (CENP)-A. Our immunoblotting experiments revealed two other chromosomal antigens recognized by ACA. These were first designated CREST-77 and CREST-110 (12), but subsequent refinement of our electrophoretic procedures and the finding that the antibodies are present in nonCREST patients has led us to rename these species CENP-B (80,000 mol wt) and CENP-C (140,000 mol wt) (13).

We now report the analysis by immunoblotting of ACApositive sera from 39 patients, ACA-negative sera from 101 patients with similar diseases and syndromes, and 22 sera from normal controls. We have detected autoantibodies recognizing the three CENP antigens only in sera from the ACA-positive patients. Anti-CENP-B was present in every ACA-positive serum examined.

\section{Methods}

Patients. 140 patients were studied. All had been seen as outpatients in the University of Connecticut Health Center Hospital Clinic or as inpatients in the University of Connecticut University Hospital by fellows and faculty of the Division of Rheumatic Diseases. We have included all 39 patients shown by indirect immunofluorescence to have ACA. 101 additional patients were selected using the Division's computerized diagnostic record system because they had Raynaud's disease or Raynaud's syndrome plus an associated connective tissue disease. These individuals included all patients with scleroderma, CREST, and Raynaud's disease. Detailed records on all patients are available in the Division files. When inadequate sera were available as frozen samples, patients were recalled, a history and physical examination performed, and additional blood obtained. The diagnosis of systemic lupus erythematosus (SLE) was based on the American Rheumatism Association (ARA) revised criteria for the classification of SLE (17). The diagnosis of scleroderma was based on the ARA preliminary criteria for the classification of systemic sclerosis (18) (all with scleroderma proximal to the metacarpalphalangeal joints). The diagnosis of calcinosis (C) was based on the X-ray evidence of calcinosis, esophageal dysmotility (E) was based on barium swallow abnormalities in the lower third of the esophagus, sclerodactyly (S) was considered to be present when the skin of the fingers was thickened diffusely distal to the metacarpalphalangeal joint, and telangiectasiae $(T)$ was based on the presence of typical telangiectasiae on lip, fingers, hands, or multiple mat-like telangiectasias of the face. The diagnosis of Raynaud's phenomenon ( $R$ ) was based on either a history or observation of a biphasic or triphasic response to cold; Raynaud's disease was considered to be present when the patient had no 
objective evidence of another disease. The diagnosis of polymyositis was based on the criteria of Bohan and Peter for probable or definite disease (19); sicca complex was defined as the presence of at least two of the three clinical features of the sicca complex: xerophthalmia, xerostomia, or salivary gland enlargement; and the diagnosis of rheumatoid arthritis was made in patients who fulfilled the ARA criteria for the diagnosis of classic rheumatoid arthritis (20).

Sera from 22 age-, sex-, and race-matched normal individuals were used as controls. 10 of these controls were over the age of 50 .

Anti-centromere antibodies. ACA were detected using indirect immunofluorescence with commercially prepared HEp-2 cells (Wampole Laboratories, Cranbury, NJ) as substrate, and fluorescein-labeled rabbit anti-human Ig (Wampole Laboratories). Slides were viewed as previously described (21). ACA were considered to be present when the distinctive speckled pattern in metaphase was observed $(3,9)$. Each serum sample was screened for the presence of ACA at a 1:40 dilution in phosphate buffered saline ( $\mathrm{pH} 7.3 \pm 0.2)$ and further serial twofold dilutions were performed if ACA were present. Positive sera were then also tested at a titer of 1:40 using fluorescein-labeled goat anti-human $\operatorname{IgG} \gamma$-chainspecific, (Kallestad Laboratories, Inc., Austin, TX), fluorescein-labeled rabbit anti-human IgM $\mu$-chain-specific, (Accurate Chemical and Scientific Corp., Westbury, NY), and fluorescein-labeled goat anti-human IgA $\alpha$-chain-specific (Cooper Biomedical, Freehold, NJ). Sera were coded and read twice by two independent observers without knowledge of clinical status or previous ACA results. Complement fixation by ACA was tested by incubation at room temperature of $\mathrm{HEp}-2$ cells with test serum diluted 1:160 and heat-inactivated for $30 \mathrm{~min}$ at $56^{\circ} \mathrm{C}$. After washing twice for $10 \mathrm{~min}$, fresh human serum was added as a source of complement and allowed to incubate for $30 \mathrm{~min}$ at room temperature. After washing twice for $10 \mathrm{~min}$, fluorescein-conjugated goat anti-human C3 (Kallestad Laboratories, Inc.) was added and allowed to incubate for 30 min, after which the slide was washed twice and then processed as previously described (19).

Antinuclear antibodies (ANA). Antinuclear antibodies were detected by indirect immunofluorescence, using fluorescein-conjugated anti-human Ig with cryostat cut mouse liver as substrate as previously described (21). For ANA testing, undiluted sera were initially tested and, if positive, twofold dilutions were carried out.

Detection of ACA polypeptide antigens. Chromosomes were isolated from colcemid-arrested HeLa cells and subjected to SDS-PAGE in 12.5\% gels as described by Lewis and Laemmli (22). The separated proteins were electrophoretically transferred to nitrocellulose paper under standard conditions (23) except that the transfer buffer contained $0.1 \%$ SDS. The nitrocellulose blot was cut into 20-30 thin longitudinal strips. Each of these was numbered and incubated with $5 \mathrm{ml}$ of buffer containing 4\% bovine serum albumin (Pentex grade; Miles Laboratories, Naperville, IL) as a blocking agent (12). After 30 min the patient's serum was added to give the following final dilutions: 1:150 to 1:250 for sera which were ACA-negative or ACA-positive with a titer of 1:640 or less; 1:400 for sera with ACA of 1:1280; and 1:500 for ACA-positive sera with titers of $>1: 1280$. For certain ACA-positive sera, dilutions of 1:30, 1:50, or 1:100 were also used. After incubation overnight at room temperature, the serum was removed, the strips washed, and the bound human antibody detected with ${ }^{125}$ I-protein A (from Staphylococcus aureus, Pharmacia Fine Chemicals AB, Uppsala, Sweden) and subsequent autoradiography as previously described $(12,23)$.

\section{Results}

Patients. The diagnoses of the 140 patients are shown in Tables I and II.

ACA-positive patients. There was 1 male and 38 females; all were Caucasian except for 1 Puerto Rican female and 1 Black female. Ages ranged from 27 to 79 with a mean of $50.87 \pm 11.18$ yr. The disease duration ranged from 2 mo in a patient with Raynaud's disease to $32 \mathrm{yr}$ in a patient with CREST and sicca complex. All patients had Raynaud's syndrome or disease (Table
Table I. Diagnosis of Patients

\begin{tabular}{lcc}
\hline & No. of patients & ACA \\
\hline CREST* $^{*}{ }^{*}$ With scleroderma & 65 & 27 \\
$\quad$ Without scleroderma & 23 & 11 \\
Proximal scleroderma without CREST & 42 & 16 \\
Raynaud's disease & 12 & 0 \\
SLE with Raynaud's & 36 & 7 \\
Sicca with Raynaud's & 12 & 1 \\
Raynaud's with arthritis & 13 & 3 \\
& 2 & 1 \\
& Total 140 & 39 \\
\hline
\end{tabular}

* Defined as Raynaud's plus C, E, S, or T

$\ddagger$ Proximal scleroderma

I). CREST syndrome was the second most common finding (27 patients). Of the CREST patients, complete CREST was present in 12 and partial CREST in 15 (defined as Raynaud's syndrome plus at least one of the following: calcinosis, telangiectasia, esophageal dysmotility, sclerodactyly). Proximal scleroderma was present in 11 of the 27 CREST patients. More than one diagnosis was found in many patients. The detailed diagnoses of the ACApositive patients are presented in Table II.

Anticentromere antibodies. ACA titers using indirect immunofluorescence ranged from 1:320 to 1:20,480 (Fig. 1). Sera from the 22 normal controls and the 101 patient controls were negative. The titer of ACA was unrelated to the presence or absence of CREST, sicca complex, or any other disease or specific manifestation. Titers in the patients with Raynaud's disease were not lower than those with complete or partial CREST.

IgM and IgA ACA were not detected. Complement fixing IgG ACA were present in 17 of the 39 patients. Presence of complement fixing ACA did not correlate with specific diseases, manifestations, or with disease duration.

Table II. Summary of Clinical Findings for ACA-positive Patients

Raynaud's without (C, E, S, or T)

plus sicca complex

plus SLE

plus arthritis

Raynaud's plus (C, E, S, or T)§ 7

plus proximal scleroderma 4

plus lung $\quad 5$

plus SLE 1

plus sicca complex 3

plus rheumatoid arthritis (RA) 1

plus lung and gastrointestinal abnormalities 1

plus SLE 1

plus RA 1

plus polymyositis, RA, and lung 1

plus Hashimoto's thyroiditis 1

* In Fig. 1 corresponds to Raynaud's only and nonCREST. $\ddagger$ One patient with vasculitis and heterozygous $\mathrm{C}_{4}$ deficiency. $\S$ In Fig. 1 corresponds to CREST and partial CREST. 


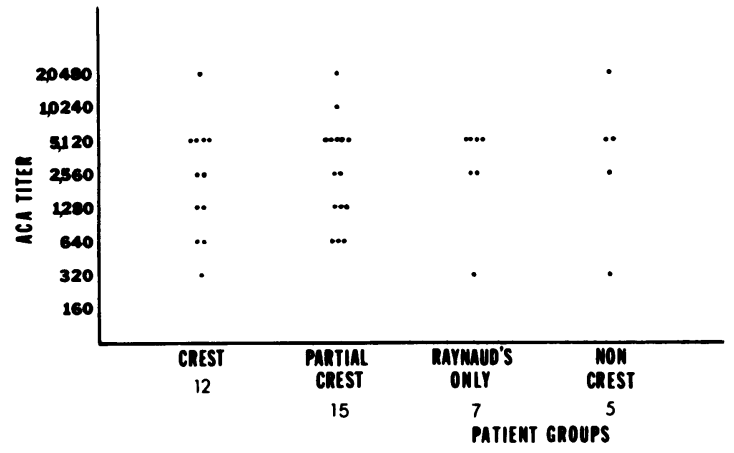

Figure 1. Titers of ACA in 39 patients.

Persistence of specific anti-centromere antibodies. More than one serum sample was tested on 15 patients ( 2 samples in 11; 3 samples in 3, and 7 samples in 1 patient). The disease duration between the first and last sample studied from each patient ranged from 1 mo to $9 \mathrm{yr}$ and was more than $2 \mathrm{yr}$ in 10 of the 15 patients. There was no change in antigens recognized over time by sequential serum samples for any patient.

Relation between the presence of $A N A$ and $A C A$. ANA was tested in 38 of the 39 patients. ANA was negative using undiluted serum in 2 patients, titers $<1: 16$ were found in 16 patients, and titers of $1: 32$ to $1: 128$ were found in 20 patients. There was no relationship between the ACA titer and the ANA titer using mouse liver as substrate. The two ANA-negative sera had ACA titers of $1: 320$ and $1: 1280$.

Antigens detected by $A C A$. One centromere protein antigen was recognized by sera from all 39 ACA-positive patients, and two other centromere antigens were recognized by 36 of the 39 . These antigens are: CENP-A (17,000 mol wt, 38 of 39 patients), CENP-B (80,000 mol wt, 39 of 39 patients), and CENP-C (140,000 mol wt, 37 of 39 patients). The results of a typical screening experiment are shown in Fig. 2. Certain sera exhibit lower titers of antibodies recognizing CENPs A and/or C (Fig. 2 , lanes $a, e, k, l$, and $u$ ). When these sera were tested at lower dilutions (i.e., to 1:30-1:50), in all but three cases the missing antibody species could be detected. However, at very low serum dilutions, the nonspecific background on the blots increases substantially with the consequence that small amounts of antibodies binding to CENPs $\mathrm{A}$ and $\mathrm{C}$ are obscured. We therefore suspect that very low levels of antibodies recognizing CENPs $A$ and $C$ are present in the three sera where we have as yet been unable to demonstrate their presence, but cannot prove this with our current techniques.

Four of the 39 ACA-positive sera contain antibodies recognizing a group of antigens which migrate in SDS-PAGE with an $M_{\mathrm{r}}$ of 21-25,000 (Fig. 2, lane $m$ ). Affinity-purified antibodies recognizing these antigens are inactive in immunofluorescence $(13,14)$ and therefore the chromosomal location of these antigens is unknown.

Fig. 2 also shows that some antibody binds to $\mathrm{H} 1$ and $\mathrm{H} 3$ under our conditions. We believe that the bulk of this binding is nonspecific for the following reasons. Approximately one-third of the total weight of the chromosome is histone. Therefore these proteins are present in vast molar excess over all other antigens in the immunoblots. When proteins of whole cells (where histones are relatively minor) are probed by blotting, the CENPs are detected, but binding to histones is not observed (13).

We have previously affinity-purified antibodies which bound to the $\mathrm{H} 1$ region of immunoblots (13). In contrast to antibodies binding to the CENP antigens, antibodies eluted from $\mathrm{H} 1$ do not rebind to this species when used to reprobe blots of chromosomal proteins (13). We conclude that the binding to $\mathrm{H} 1$ is not specific.

Binding to histone $\mathrm{H} 3$ is also apparently nonspecific. Antibodies which have been affinity-eluted from other regions of preparative immunoblots under highly denaturing conditions (3 $\mathrm{M} \mathrm{NH}_{4}$-thiocyanate, $\mathrm{pH} 11.5$, reference 13) exhibit a dramatic increase in rebinding to the $\mathrm{H} 3$ region with increasing time of exposure to denaturant (Saunders, W., unpublished data). This suggests that denatured antibodies bind to $\mathrm{H} 3$ in our blot system. We cannot exclude the possibility that some of these patients do have low titer antibodies recognizing H3. In fact, the possibility for weak cross reaction of ACA with histones is suggested by preliminary DNA sequence data which indicates that CENP$B$ may have several locally concentrated regions of basic amino

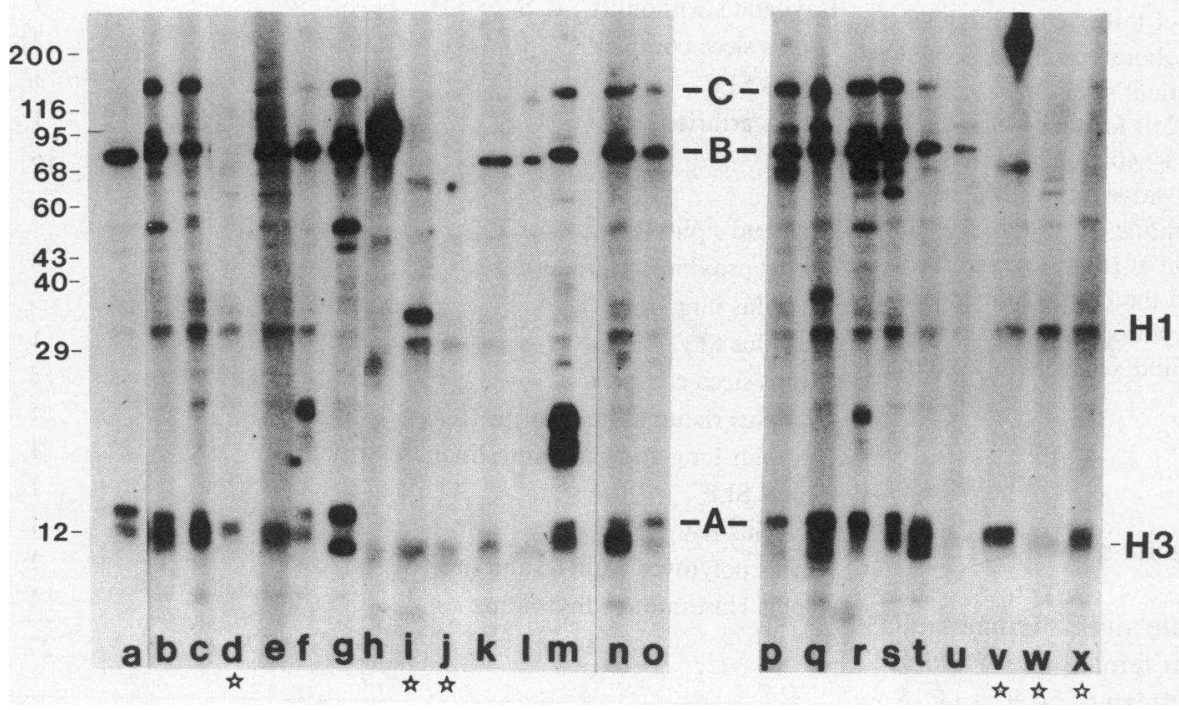
carbonic anhydrase $(29,000 \mathrm{~mol} \mathrm{wt})$; and cytochrome $\mathrm{C}(12,000 \mathrm{~mol} \mathrm{wt})$.
Figure 2. Results of a typical experiment screening sera from 17 ACA-positive patients, 6 ACA-negative patients (lanes $h, i, j$, $v, w, x$ ), and 1 normal control (lane $d$ ). ACA-negative lanes are indicated by an * below the figure. The antigen was a nitrocellulose blot of human chromosomal proteins which was cut into longitudinal strips and probed with the various sera as described in Methods. The locations of CENP-A (17,000 mol wt), CENP-B (80,000 mol wt), and CENP-C (140,000 mol wt) are indicated between lanes $o$ and $p$. The position of histones $\mathrm{H} 1$ and $\mathrm{H} 3$ (determined by staining the blot with Ponceau $S$ before antibody treatment) are indicated to the right of the blot. The mobilities of markers used in independent experiments to determine the relative molecular weights of the CENP antigens are indicated to the left of lane $a$. The markers are: myosin $(200,000 \mathrm{~mol} \mathrm{wt})$ beta-galactosidase $(116,000 \mathrm{~mol} \mathrm{wt})$; phos- 
acids (Sullivan, K., D. Cleveland, N. Rothfield, and W. Earnshaw, unpublished data).

\section{Discussion}

This paper examines the specificity of the ACA response. We wish to know whether all individuals with ACA have undergone a similar autoimmune process, or whether numerous different pathways may lead to the production of ACA. In the former case it makes sense to examine the relationship between specific autoantibodies and defined diseases or manifestations, while in the latter case such an analysis is probably impossible.

The criterion which we will use to define the specificity of the autoimmune response is the exact pattern of chromosomal antigens recognized by each patient serum. Because ACA occur with low frequency in the population, most studies of centromeric autoantigens have involved independent research groups with access to limited numbers of patient sera. In the studies of which we are aware, immunoblot data have been published using a total of 22 patients sera: $1,(11) ; 1,(15) ; 1,(16) ; 18,(14)$; and $1,(24)$. We have extended this work by analyzing 39 additional patient sera under conditions designed to optimize the detection of insoluble antigens. As we discuss below, this large scale study has enabled us to conclude that all members of an ACA-positive patient population have apparently undergone a remarkably similar autoimmune process.

Clinical findings. All ACA-positive patients in our study have Raynaud's syndrome or disease. Thus, although ACA were first described in patients with CREST syndrome (1-3), the specific clinical finding which best correlates with the presence of ACA is Raynaud's phenomenon. In this and studies by three other groups, the correlation between Raynaud's phenomenon and ACA remains at $100 \%$. The other studies included 14 (3), 27 (5), and 35 (2) ACA-positive individuals. The only dissenting data come from one study of patients with primary biliary cirrhosis (9) where two of ten ACA-positive patients did not have Raynaud's syndrome. The low incidence of ACA makes the study of large numbers of patients difficult for any one research group, but the association between ACA and Raynaud's phenomenon is dramatic when the findings from the five studies are combined $(123 / 125$ or $98 \%)$.

Patients with isolated Raynaud's disease also occasionally have ACA. The incidence among our patients $(7 / 36$ or $19 \%)$ was very like that found earlier by Fritzler et al. ( $21 \%$, reference 2), although a third study of 38 patients failed to reveal any ACA-positive individuals (4).

In four of the patients studied by Fritzler et al. (2), Raynaud's phenomenon (and ACA) preceded development of the CREST syndrome. This does not mean, however, that Raynaud's disease is an obligatory precursor to the subsequent development of CREST or scleroderma. Three of our seven ACA-positive Raynaud's disease patients have had their disease and nothing else for $\mathbf{2 0}$ or more years. In addition, five ACA-positive Raynaud's disease patients developed no additional clinical manifestations during a 2-yr prospective study (5).

It will be important to follow patients with isolated Raynaud's disease for prolonged periods in order to determine the frequency with which the disease is either a precursor or the first manifestation of systemic connective tissue disease. At the present time it seems premature to predict a poor prognosis for ACA-positive Raynaud's disease patients.

Centromeric autoantigens. Our results indicate that all individuals with ACA have apparently undergone a very similar autoimmune response. When proteins of isolated human chromosomes are resolved by SDS-PAGE and probed by immunoblotting with ACA-positive sera, a protein with an $M_{\mathrm{r}}$ of 80,000 (CENP-B) is recognized. Most ACA-positive sera also recognize two other polypeptides; CENP-A (17,000 mol wt) and CENP$\mathrm{C}(140,000 \mathrm{~mol} \mathrm{wt})$, though occasionally these antibodies are present at much lower titers than anti-CENP-B (in three sera one or the other of these antibodies was undetectable). Antibodies recognizing the three CENP antigens appeared coordinately in the serum of a patient who became ACA-positive concomitant with development of Raynaud's syndrome (10, 13). We have yet to observe antibodies recognizing the CENP species in any ACA-negative serum, either from patients or from normal controls.

We have also recognized antibodies to the CENP species in ACA-positive sera from other institutions. These include gifts of two sera from Dr. E. Tan (Scripps Clinic and Research Foundation, LaJolla, CA), two sera from Dr. W. van Venrooij (University of Nijmegen, Nijmegen, The Netherlands), three sera from Dr. H. Guldner (University of Heidelberg, Heidelberg, Federal Republic of Germany), one serum from Dr. H. Langevin (Johns Hopkins Hospital, Baltimore, MD), and one serum from Dr. R. Partridge (Harvard Medical School, Boston, MA).

Other workers have generally failed to detect anti-CENPs B and $C$ by immunoblotting $(11,14-16,24)$. Only CENP-A has been recognized $(11,14,24)$. We suggest four possible reasons why the major centromere autoantigen could be missed by the immunoblotting procedure. $(a)$ In one study, the antigens were apparently detected, but were not discussed (11). (b) CENPs B and $C$, which are highly insoluble, may be discarded by certain subcellular fractionation protocols, which are designed to yield soluble nuclear antigens $(14,15)$. (c) The antigens may transfer poorly under certain conditions of immunoblotting. Problems with electrophoretic transfer of antigens from the gel to the nitrocellulose have been cited in one of the studies (24). (d) CENPs $B$ and $C$ may be absent from commercial antigen preparations as a result of proteolysis $(15,16,24)$.

Whatever the explanation, it should be emphasized that CENP-A cannot be the sole centromere antigen as suggested by others $(14,24)$, since this antigen is quantitatively absent from mitotic chromosome scaffolds (12). These structures still exhibit strong centromeric staining with ACA (12).

The relationship between CENPs A, B, and C is complex. While CENP-B shares epitopes with both $A$ and $C$, these apparently represent nonoverlapping sites on the protein, as we have not yet detected any epitopes shared between CENPs A and $C(13)$. This suggests that CENP-B could be the primary autoantigen, with binding to CENPs $A$ and $C$ resulting from fortuitous cross reaction. The observation that anti-CENPs $A$ and $C$ are present at variable titers, while the titer of anti-CENP$B$ is uniformly high, is also consistent with the idea that $B$ might be the primary autoantigen. It is currently not known whether CENPs $A, B$, and $C$ represent the products of a single gene, a multi-gene family, or of unrelated genes. Molecular cloning experiments designed to answer this question are currently in progress (26).

Whatever the relationship between CENPs A, B, and C, these results indicate that antibody to CENP-B is diagnostic for the presence of ACA. For laboratories which routinely perform SDSPAGE, immunoblotting may well be the primary screening method of choice. Immunoblotting is not performed in most clinical laboratories, however, and we are currently developing a simple enzyme-linked immunosorbent assay (ELISA) test using 
cloned CENP-B (26). Availability of such an ELISA will make it feasible to screen large patient and control populations for this antibody.

We have shown above that the ACA response is directed against the CENP antigens. But as we have previously shown, these are very minor cellular components (12). How could such minor proteins become targets of a major immune response which can lead to circulating antibody titers of up to $1: 20,800$ ? This is the subject of much current debate (27). In order to approach this question, it will be necessary to obtain specific probes recognizing each CENP antigen, and to map the distribution of these antigens in patients and in controls.

Given the $100 \%$ correlation between ACA and Raynaud's phenomenon, the logical place to look for the CENP antigens in vivo is in smooth muscle and/or endothelial cells. The antigens might be displayed at the cell surface as a result of damage, as has been recently demonstrated for the Ro, RNP, and Sm antigens of keratinocytes (28). Conversely, the circulating antibody may cause damage to endothelial cells.

We believe that the highly specific and well characterized nature of the ACA response makes this system an ideal one for examination of possible relationships between the presence of specific autoantibodies and defined disease manifestations.

Note added in proof. CENPs A and B, but not CENP-C, have recently been detected in immunoblots of human chromosomes. (Valdivia, M. M., and B. R. Brinkley. 1985. J. Cell Biol. 101:1124-1134.).

\section{Acknowledgments}

We thank Drs. E. Tan, W. van Venrooij, H. Guldner, H. Langevin, and R. Partridge for the gift of sera and Dr. J. Korn for helpful discussions.

This work was supported by National Institutes of Health grants RO1 GM30985 and P60 AM20621 and a grant from the University of Connecticut Research Foundation.

\section{References}

1. Moroi, Y., C. Peebles, M. J. Fritzler, J. Steigerwald, and E. M. Tan. 1980. Autoantibody to centromere (kinetochore) in scleroderma sera. Proc. Natl. Acad. Sci. USA. 77:1627-1631.

2. Fritzler, M. J., T. D. Kinsella, and E. Garbutt. 1980. The CREST syndrome: a distinct serologic entity with anticentromere antibodies. Am. J. Med. 69:520-526.

3. Tan, E. M., G. P. Rodnan, I. Garcia, Y. Moroi, M. J. Fritzler, and C. Peebles. 1980. Diversity of antinuclear antibodies in progressive systemic sclerosis: anti-centromere antibody and its relationship to CREST syndrome. Arthritis Rheum. 23:617-625.

4. Kallenberg, C. G. M., G. W. Pastoor, A. A. Wouda, and T. H. The. 1982. Antinuclear antibodies in patients with Raynaud's phenomenon: clinical significance of anticentromere antibodies. Ann. Rheum. Dis. 41:382-387.

5. Catoggio, L. J., R. M. Bernstein, C. M. Black, G. R. V. Hughes, and P. J. Maddison. 1983. Serological markers in progressive systemic sclerosis: clinical correlations. Ann. Rheum. Dis. 42:23-27.

6. Garcia-de la Torre, I., and L. Miranda-Mendez. 1982. Studies of antinuclear antibodies in rheumatoid arthritis. J. Rheumatol. 9:603606.

7. Seibold, J. R., and M. C. Trontell. 1982. Anticentromere antibody and primary pulmonary hypertension. J. Rheumatol. 9:607-609.

8. Makinen, D., M. Fritzler, P. Davis, and S. Sherlock. 1983. Anticentromere antibodies in primary biliary cirrhosis. Arthritis Rheum. 26:914-917.
9. Bernstein, R. M., M. E. Callender, J. M. Neuberger, G. R. V. Hughes, and R. Williams. 1982. Anticentromere antibody in primary biliary cirrhosis. Ann. Rheum. Dis. 41:612-614.

10. Tramposch, H. D., C. D. Smith, J.-L. Senecal, and N. Rothfield. 1984. A long-term longitudinal study of anticentromere antibodies. $\mathrm{Ar}$ thritis Rheum. 27:121-124.

11. Cox, J. V., E. A. Schenk, and J. B. Omsted. 1983. Human anticentromere antibodies: distribution, characterization of antigens and effect on microtubules organization. Cell. 35:331-339.

12. Earnshaw, W. C., N. Halligan, C. Cooke, and N. Rothfield. 1984 The kinetochore is part of the metaphase chromosome scaffold. J. Cell Biol. 98:352-357.

13. Earnshaw, W. C., and N. F. Rothfield. 1985. Identification of a family of human centromere proteins using autoimmune sera from patients with scleroderma. Chromosoma (Berl.). 91:313-321.

14. Guldner, H. H., H.-J. Lakomek, and F. A. Bautz. 1985. Human anti-centromere sera recognize a $19.5 \mathrm{kD}$ nonhistone chromosomal protein from HeLa cells. Clin. Exp. Immunol. 58:13-20.

15. Ayer, L. M., and M. J. Fritzler. 1984. Anticentromere antibodies bind to trout testis hormone 1 and a low molecular weight protein from rabbit thymus. Mol. Immunol. 21:761-770.

16. Nishikai, M., Y. Okano, H. Yamashita, and M. Watanabe. 1984. Characterisation of centromere (kinetochore) antigen reactive with sera of patients with a scleroderma variant (CREST syndrome). Ann. Rheum. Dis. 43:819-824.

17. Tan, E. M., A. S. Cohen, J. F. Fries, A. T. Masi, D. J. McShane, N. F. Rothfield, J. G. Schaller, N. Talal, and R. J. Winchester. 1982. The 1982 revised criteria for the classification of systemic lupus erythematosus. Arthritis Rheum. 25:1271-1277.

18. Subcommittee for scleroderma criteria of the ARA diagnostic and therapeutic criteria committee. 1980. Preliminary criteria for the classification of systemic sclerosis (scleroderma). Arthritis Rheum. 23:581-590.

19. Bohan, A., and J. B. Peter. 1975. Polymyositis and dermatomyositis. N. Engl. J. Med. 292:344-407.

20. Ropes, M. W., G. A. Bennett, S. Caleb, R. Jacox, and R. A. Jessar. 1958. 1958 Revision of diagnostic criteria for rheumatoid arthritis. Bull. Rheum. Dis. 9:175-176.

21. Schrager, M. A., and N. F. Rothfield. 1976. Clinical significance of serum properdin levels and properdin deposition in the dermal epidermal junction in systemic lupus erythematosus. J. Clin. Invest. 57:212-221.

22. Lewis, C. D., and U. K. Laemmli. 1982. Higher-order metaphase chromosome structure: evidence for metalloprotein interactions. Cell. 29:171-181.

23. Towbin, H., T. Stahelin, and J. Gordon. 1979. Electrophoretic transfer of proteins from polyacrylamide gels to nitrocellulose sheets: procedure and some applications. Proc. Natl. Acad. Sci. USA. 7:43504354.

24. van Venrooij, W. J., S. O. Stapel, H. Houben, W. J. Habets, C. G. M. Kallenberg, E. Penner, and L. B. van de Putte. 1985. Scl-86, a marker antigen for diffuse scleroderma. J. Clin. Invest. 75:1053-1060.

25. McCarty, G. A., J. R. Rice, M. L. Bembe, and F. A. Barada. 1983. Anticentromere antibody-clinical correlations and association with favorable prognosis in patients with scleroderma variants. Arthritis Rheum. 26:1-7.

26. Earnshaw, W. C., K. F. Sullivan, W. S. Saunders, D. W. Cleveland, and N. F. Rothfield. 1985. Identification and molecular cloning of CENP$B$, a human centromere protein recognized by all human anticentromere antibodies (ACA). Clin. Res. 33:591a. (Abstr.)

27. Schoenfeld, Y., and R. S. Schwartz. 1984. Immunologic and genetic factors in autoimmune diseases. N. Engl. J. Med. 311:10191029.

28. LeFeber, W. P., D. A. Norris, S. R. Ryan, J. C. Huff, L. A. Lee, M. Kubo, S. T. Boce, B. L. Kotzin, and W. L. Weston. 1984. Ultraviolet light induces binding of antibodies to selected nuclear antigens on cultured human keratinocytes. J. Clin. Invest. 74:1545-1551. 